TRANSACTIONS OF THE

AMERICAN MATHEMATICAL SOCIETY

Volume 357, Number 9 , Pages 3459-3479

S 0002-9947(05)03944-9

Article electronically published on April 27, 2005

\title{
HOW TO OBTAIN TRANSIENCE FROM BOUNDED RADIAL MEAN CURVATURE
}

\author{
STEEN MARKVORSEN AND VICENTE PALMER
}

\begin{abstract}
We show that Brownian motion on any unbounded submanifold $P$ in an ambient manifold $N$ with a pole $p$ is transient if the following conditions are satisfied: The $p$-radial mean curvatures of $P$ are sufficiently small outside a compact set and the $p$-radial sectional curvatures of $N$ are sufficiently negative. The 'sufficiency' conditions are obtained via comparison with explicit transience criteria for radially drifted Brownian motion in warped product model spaces.
\end{abstract}

\section{Introduction}

A Riemannian manifold is called transient if the Brownian motion from a point in the manifold does not hit every precompact open subset with probability 1 . The notion of transience has several equivalent analytic formulations in potentialtheoretic terms. The Kelvin-Nevanlinna-Royden Criteria (see [LS]) assures for example that a manifold is transient if and only if its Laplace operator admits a positive fundamental Green kernel; transience is thus equivalent to the notion of hyperbolicity. Yet another equivalent condition for transience is the existence of a compact subset with strictly positive capacity. This latter criterion will be used throughout this paper.

From a geometric point of view an interesting quest in this setting is the one alluded to in the title. Suppose $P$ is a submanifold in an ambient Riemannian manifold $N$. The problem is then to find the most general intrinsic geometric condition on $N$ and the most general extrinsic geometric condition on $P$ which together will assure that $P$ is transient. In [MP2] we proved transience of minimal submanifolds of dimension $m \geq 3$ in Hadamard-Cartan manifolds. Further, when $m=2$ we still get transience under the assumption of minimality, but then only under the slightly stronger condition that the ambient Hadamard-Cartan manifold has strictly negative sectional curvatures.

In the present paper we extend this result in essentially two ways.

Received by the editors October 10, 2003.

2000 Mathematics Subject Classification. Primary 53C17, 31C12, $60 \mathrm{~J} 65$.

Key words and phrases. Transience, capacity, drifted Brownian motion, submanifolds, mean curvature, radial mean curvature, warped products, model spaces, Hadamard-Cartan manifolds, extrinsic annuli, comparison theory.

The first author was supported by the Danish Natural Science Research Council.

The second author was supported by DGI grant No. BFM2001-3548 and the Danish Natural Science Research Council.

(C)2005 American Mathematical Society Reverts to public domain 28 years from publication 
1.1. Concerning the ambient space. First, we allow the ambient space $N^{n}$ to have some positive sectional curvatures, although it should still possess at least one pole. A pole is a point $p$ from where the exponential map is a diffeomorphism: $\exp _{p}: T_{p} N^{n} \rightarrow N^{n}$. For comparison, a Hadamard-Cartan manifold has everywhere non-positive sectional curvatures, and since it is also by definition simply connected, every point is a pole. The rôle of the pole $p$ is precisely to serve as the origin of a smooth distance function $r$ from $p$ : For every $x \in N^{n}-\{p\}$ we define $r(x)=\operatorname{dist}_{N}(p, x)$, and this distance is realized by a unique geodesic from $p$ to $x$. The sectional curvatures of $N$ along these geodesics are called the $p$-radial sectional curvatures of $N$ and are denoted by $K_{p, N}$. They control the second-order behavior of $r(x)$ via the classical Jacobi field index theory. Indeed, a bound on the $p$-radial sectional curvatures gives a bound on the Hessian of radial functions, $\operatorname{Hess}^{N}(f(r))$, as proved by Greene and $\mathrm{Wu}$; see [GreW].

1.2. Concerning the submanifold. The submanifold $P$ and the restricted radial functions $\left.f(r)\right|_{P}$ inherit this second order bound to the $P$-intrinsic Hessian, Hess $\left.^{P} f(r)\right|_{P}$, and therefore also to the Laplacian $\left.\Delta^{P} f(r)\right|_{P}$ of such modified distance functions. However, the mean curvatures $H_{P}$ of $P$ also appear in this Laplacian via the $p$-radial mean curvature function for $P$ in $N$, which is defined in terms of the inner product of $H_{P}$ with the $N$-gradient of the distance function $r(x)$ as follows:

$$
H_{p, P}(x)=-\left\langle\nabla^{N} r(x), H_{P}(x)\right\rangle \quad, \quad x \in P .
$$

A suitable control on the p-radial mean curvatures together with a suitable control on the $p$-radial sectional curvatures of the ambient space will therefore eventually control the Laplacian of restricted radial functions on $P$. In particular we consider potential functions stemming from similar capacity calculations of radially symmetric comparison spaces and transplant them to $P$ via the distance function $r$ in $N$. Such transplantations are then compared with the 'correct' potentials on $P$, and the maximum principle for the Laplacian $\Delta^{P}$ finally gives the comparison result for capacities in $P$. Thus we have the second extension of our previous work [MP2] in this direction: Here the submanifolds $P$ in $N$ are allowed to have non-vanishing (although still bounded) mean curvatures. The precise bound on the mean curvatures is formulated in terms of a radial function $\mathcal{H}(r)$. The exponential integral of this function plays a direct rôle in our sufficient condition for transience; see Theorem A below.

These remarks and tools will, of course, be substantially specified and precisely defined in the following sections. So far they do permit, however, a first direct statement of our main theorem and some of its corollaries.

Theorem A. Let $P^{m}$ denote an unbounded submanifold of an ambient manifold $N^{n}$ with a pole $p$. Let $w(r)$ denote a positive function of $r=r(x)=\operatorname{dist}_{N}(p, x)$ such that $w(0)=0$ and $w^{\prime}(0)=1$.

Suppose that the p-radial mean curvatures of $P$ are bounded by a continuous radial function $\mathcal{H}(r)$ as follows:

$$
-\left\langle H_{P}(x), \nabla^{N} r(x)\right\rangle \leq \mathcal{H}(r) \leq \frac{w^{\prime}(r)}{w(r)} \quad \text { for all } x \text { with } r=r(x)>\rho .
$$


Suppose that the p-radial sectional curvatures of $N$ are bounded by a radial function $\mathcal{K}(r)$ as follows:

$$
K_{p, N}\left(\sigma_{x}\right) \leq \mathcal{K}(r) \leq-\frac{w^{\prime \prime}(r)}{w(r)} \quad \text { for all } x \text { with } r=r(x)>0
$$

Finally, suppose further that

$$
\int_{\rho}^{\infty} \frac{\mathcal{G}^{m}(r)}{w^{m-1}(r)} d r<\infty, \text { where } \mathcal{G}(r)=\exp \left(\int_{\rho}^{r} \mathcal{H}(t) d t\right) .
$$

Then $P^{m}$ is transient.

For convenience and for transparency we show in Sections 2 and 6 below how the $w$-controlled conditions in Theorem A stem from the analysis of a suitable drifted modification of the Laplacian on so-called model spaces. A $w$-model space $M_{w}^{m}$ is a warped product of a real interval with the standard $(m-1)$-dimensional unit sphere and warping function $w(r)$. For each positive function $w(r)$ the corresponding $w$-model space will serve as both explicit geometric origin and background for the comparison construction, which in turn permits a geometrically convenient reformulation of the theorem. The proof of Theorem A is concluded in Section 8 In Section 9 we will prove the following consequences of the theorem, among others:

Corollary B. Let $P^{m}(m \geq 2)$ be an unbounded immersed submanifold of an Hadamard-Cartan manifold $N^{n}$ with sectional curvatures $K_{N}$ bounded from above by $b<0$. Suppose that the $p$-radial mean curvature function $H_{p, P}(x)$ of $P^{m}$ in $N^{n}$ from some given point $p$ in $N$ satisfies

$$
H_{p, P}(x) \leq \mathcal{H}_{0}<\frac{m-1}{m} \sqrt{-b}
$$

for some constant $\mathcal{H}_{0}$ and for all $x \in P^{m}$ outside some compact set. Then $P^{m}$ is transient.

Corollary E. Suppose $P^{2}$ is an unbounded 2-dimensional surface in a manifold $N^{n}$ with a pole $p$. Suppose that (outside the metric sphere of radius $\rho$ and center $p$ in $N^{n}$ ) the surface $P$ is either a p-radial cone or a minimal surface. Suppose further that there exists a constant $\varepsilon>0$, such that the p-radial sectional curvatures of $N$ are less than or equal to $-\varepsilon(1+\varepsilon) / r^{2}$ for all $r \geq \rho$ and less than or equal to $b(\varepsilon, \rho)$ for all $r \leq \rho$, where $b(\varepsilon, \rho)$ is the (negative) solution to the equation

$$
\operatorname{coth}(\rho \sqrt{-b(\varepsilon, \rho)})=(1+\varepsilon) /(\rho \sqrt{-b(\varepsilon, \rho)}) .
$$

Then $P^{2}$ is transient.

Remark a. These and several other corollaries (or rather their proofs) exemplify specific choices of the auxiliary function $w(r)$, which will work in the given situations and imply transience via Theorem A. It is, however, an interesting problem of independent interest to find the 'best possible' function $w(r)$ in terms of the integral in the transience test to be the smallest possible for any given pair of geometric bounding functions $\mathcal{H}(r)$ and $\mathcal{K}(r)$. Proposition $\mathbb{C}$ in Section 9 is a first attempt in this direction. It gives a set of quite general explicit conditions on the radial bounding functions which imply transience without any explicitly stated reference to the auxiliary function $w(r)$. 
We must note here, as well, that our techniques may be considered as generalizations (to submanifolds) of intrinsic comparison methods applied previously by Ichihara in [1] and [2]. The problem of finding intrinsic geometric criteria for transience or recurrence has recently been studied by several authors; see e.g. $[\mathrm{H}$, Gri]. By specializing the proof of Theorem A to the purely intrinsic geometric setting (such that the submanifold is the ambient manifold itself, $P=N$ ) we obtain a proof of the following well-known result (see again Section 9):

Proposition G ([11], [12]). Let $N^{n}$ denote a complete Riemannian manifold with a pole $p$. Suppose that the p-radial sectional curvatures of $N^{n}$ are bounded from above by the $p_{w}$-radial sectional curvatures of a $w$-model space $M_{w}^{m}$ for all $r>0$. Suppose furthermore that the following condition holds:

$$
\int_{\rho}^{\infty} \frac{1}{w^{n-1}(r)} d r<\infty
$$

Then $N^{n}$ is transient.

1.3. Outline of the paper. We devote Section 2 to a discussion of those aspects of warped products and model spaces which will be instrumental for our comparison analysis. The general setup for the comparison techniques is then constructed in Section 3 The basic comparison inequalities for the Laplacian are reviewed in Section 4 and in Sections 5 and 6 we define the capacity of general domains and explicitly calculate the modified (drifted) capacity of radially symmetric domains in model spaces. A first glimpse of the ensuing comparison result is given in Example 6.4. The local version of our main result is then established in Section 7 Finally the proofs of Theorem A and its corollaries are presented in Sections 8 and 9 respectively.

\section{WARPED PRODUCTS AND MODEL SPACES}

Warped products are generalized manifolds of revolution; see e.g. $\mathrm{O}^{\prime} \mathrm{N}$. Let $\left(B^{k}, g_{B}\right)$ and $\left(F^{l}, g_{F}\right)$ denote two Riemannian manifolds and let $w: B \rightarrow \mathbb{R}_{+}$be a positive real function on $B$. We assume throughout that $w(r)$ is at least $C^{1}$ with piecewise continuous second order derivative $w^{\prime \prime}(r)$. The product manifold $M^{k+l}=B \times F$ is then considered. We denote the projections onto the factors by $\pi: M \rightarrow B$ and $\sigma: M \rightarrow F$, respectively. The metric $g$ on $M$ is then defined by the following $w$-modified (warped) product metric:

$$
g=\pi^{*}\left(g_{B}\right)+(w \circ \pi)^{2} \sigma^{*}\left(g_{F}\right) .
$$

Definition 2.1. The Riemannian manifold $(M, g)=\left(B^{k} \times F^{l}, g\right)$ is called a warped product with warping function $w$, base manifold $B$ and fiber $F$. We write as follows: $M_{w}^{m}=B^{k} \times{ }_{w} F^{l}$.

Definition 2.2 (See [Gri], GreW]). A $w$-model $M_{w}^{m}$ is a smooth warped product with base $B^{1}=\left[0, \Lambda\left[\subset \mathbb{R}\right.\right.$ (where $0<\Lambda \leq \infty$ ), fiber $F^{m-1}=S_{1}^{m-1}$ (i.e. the unit $(m-1)$-sphere with standard metric), and warping function $w:\left[0, \Lambda\left[\rightarrow \mathbb{R}_{+}\right.\right.$ with $w(0)=0$ and $w^{\prime}(0)=1$. The point $p_{w}=\pi^{-1}(0)$ is called the center point of the model space. If $\Lambda=\infty$, then $p_{w}$ is a pole of $M_{w}^{m}$. 
Proposition 2.3. The simply-connected space forms $\mathbb{K}^{m}(b)$ of constant curvature $b$ are $w$-models with warping functions

$$
w(r)=Q_{b}(r)=\left\{\begin{array}{ll}
\frac{1}{\sqrt{b}} \sin (\sqrt{b} r) & \text { if } b>0 \\
r & \text { if } b=0 \\
\frac{1}{\sqrt{-b}} \sinh (\sqrt{-b} r) & \text { if } b<0
\end{array} .\right.
$$

Note that for $b>0$ the function $Q_{b}(r)$ admits a smooth extension to $r=\pi / \sqrt{b}$.

Proposition 2.4 (See e.g. ['N]). Let $M_{w}^{m}=B \times_{w} S_{1}^{m-1}$ be a $w$-model. Let $r_{0}$ and $r$ denote two values in $B$. Then the geodesic distance from every $x \in \pi^{-1}(r)$ to $\pi^{-1}\left(r_{0}\right)$ is $\left|r-r_{0}\right|$.

Proposition 2.5 (See ['N], p. 206). Let $M_{w}^{m}$ be a w-model with warping function $w(r)$ and center $p_{w}$. The distance sphere of radius $r$ and center $p_{w}$ in $M_{w}^{m}$ is the fiber $\pi^{-1}(r)$. This distance sphere has the following constant mean curvature vector in $M_{w}^{m}$ :

$$
H_{\pi^{-1}(r)}=-\eta_{w}(r) \nabla^{M} \pi=-\eta_{w}(r) \nabla^{M} r,
$$

where the mean curvature function $\eta_{w}(r)$ is defined by

$$
\eta_{w}(r)=\frac{w^{\prime}(r)}{w(r)}=\frac{d}{d r} \ln (w(r)) .
$$

In particular we have for the constant curvature space forms $\mathbb{K}^{m}(b)$ :

$$
\eta_{Q_{b}}(r)=\left\{\begin{array}{ll}
\sqrt{b} \cot (\sqrt{b} r) & \text { if } b>0 \\
1 / r & \text { if } b=0 \\
\sqrt{-b} \operatorname{coth}(\sqrt{-b} r) & \text { if } b<0
\end{array} .\right.
$$

Definition 2.6. Let $p$ be a point in a Riemannian manifold $M$ and let $x \in M-\{p\}$. The sectional curvature $K_{M}\left(\sigma_{x}\right)$ of the two-plane $\sigma_{x} \in T_{x} M$ is then called a $p$-radial sectional curvature of $M$ at $x$ if $\sigma_{x}$ contains the tangent vector to a minimal geodesic from $x$ to $p$. We denote these curvatures by $K_{p, M}\left(\sigma_{x}\right)$.

Proposition 2.7 (See [GreW] and [Gri]). Let $M_{w}^{m}$ be a w-model with center point $p_{w}$. Then the $p_{w}$-radial sectional curvatures of $M_{w}^{m}$ at every $x \in \pi^{-1}(r)$ (for $r>0$ ) are all identical and determined by

$$
K_{p_{w}, M_{w}}\left(\sigma_{x}\right)=-\frac{w^{\prime \prime}(r)}{w(r)} .
$$

Remark b. This radial curvature function of a $w$-model space is only piecewise continuous due to the weak differentiability assumptions for $w(r)$.

\section{Comparison CONStellations}

Given an immersed $m$-dimensional submanifold $P^{m}$ in a complete Riemannian manifold $N^{n}$ with a pole $p$, we denote the distance function from $p$ in the ambient space $N^{n}$ by $r(x)=\operatorname{dist}_{N}(p, x)$ for all $x \in N$. Since $p$ is a pole there is, by definition, a unique geodesic from $x$ to $p$ which realizes the distance $r(x)$. We also denote by $r$ the restriction $\left.r\right|_{P}: P \longrightarrow \mathbb{R}_{+} \cup\{0\}$. This restriction is then called the extrinsic distance function from $p$ in $P^{m}$. The corresponding extrinsic metric balls 
of (sufficiently large) radius $R$ and center $p$ are denoted by $D_{R}(p) \subseteq P$ and defined as any connected component of the intersection

$$
B_{R}(p) \cap P=\{x \in P \mid r(x)<R\} \quad,
$$

where $B_{R}(p)$ denotes the geodesic $R$-ball around the pole $p$ in $N^{n}$. The extrinsic ball $D_{R}(p)$ is then a connected domain in $P^{m}$, with boundary $\partial D_{R}(p)$. Since $P^{m}$ is assumed to be unbounded in $N$, we have for every sufficiently large $R$ that $B_{R}(p) \cap P \neq P$. Using the corresponding extrinsic balls we define the $p$-centered extrinsic annuli $A_{\rho, R}(p)$ in $P^{m}($ for $\rho<R)$ as

$$
A_{\rho, R}(p)=D_{R}(p)-D_{\rho}(p) \quad .
$$

In order to control the mean curvatures $H_{P}(x)$ of $P^{m}$ at distance $r$ from $p$ in $N^{n}$ we introduce the following two continuous functions of $r$ :

Definition 3.1. A radial bounding function for the $p$-radial mean curvature function of $P$ is any function $\mathcal{H}(r)$ which satisfies the inequality

$$
\mathcal{H}(r) \geq \max _{x \in \partial D_{r}}\left(-\left\langle\nabla^{N} r(x), H_{P}(x)\right\rangle\right) \quad .
$$

This gives rise to the following exponential integral (which will prove useful below), as already alluded to by the statement of Theorem A in the Introduction:

$$
\mathcal{G}(r)=\exp \left(\int_{\rho}^{r} \mathcal{H}(t) d t\right) \quad \text { for all } r \geq \rho .
$$

Remark c. Whenever we only need a continuous bounding function $\mathcal{H}(r)$ we may choose equality in (3.1). It is, however, preferable in certain cases to assume that $\mathcal{H}(r)$ is a differentiable upper bound for the $p$-radial mean curvature function; see e.g. Proposition $\mathrm{C}$ in Section 9

We now collect some of the previous ingredients and formulate the general framework for our transience comparison result:

Definition 3.2. Let $N^{n}$ denote a Riemannian manifold with a pole $p$ and distance function $r=r(x)=\operatorname{dist}_{N}(p, x)$. Let $P^{m}$ denote an unbounded complete submanifold in $N^{n}$. Let $M_{w}^{m}$ denote a $w$-model with warping function $w: \pi\left(M_{w}^{m}\right) \rightarrow \mathbb{R}_{+}$ and center $p_{w}$.

Then the triple $\left\{N^{n}, P^{m}, M_{w}^{m}\right\}$ is called a comparison constellation on the interval $[0, R]$ if the following two conditions are satisfied:

(a) The mean curvature bounding function $\mathcal{H}(r)$ is itself bounded from above by the mean curvatures of the model fibers at all distances $r \geq \rho$ for some $\rho<R$ :

$$
\mathcal{H}(r) \leq \eta_{w}(r)=\frac{w^{\prime}(r)}{w(r)} \quad \text { for all } r \in[\rho, R] .
$$

(b) The $p$-radial sectional curvatures of $N$ are bounded from above by the $p_{w}$-radial sectional curvatures of $M_{w}^{m}$ :

$$
K_{p, N}\left(\sigma_{x}\right) \leq-\frac{w^{\prime \prime}(r)}{w(r)} \quad \text { for all } x \text { with } r=r(x) \in[0, R] .
$$

With this notion of a comparison constellation we can now reformulate our main transience result. The proof is developed through the following sections. 
Theorem A. Let $\left\{N^{n}, P^{m}, M_{w}^{m}\right\}$ denote a comparison constellation on $[0, \infty[$ as defined in Definition [3.2. Suppose that

$$
\int_{\rho}^{\infty} \frac{\mathcal{G}^{m}(r)}{w^{m-1}(r)} d r<\infty
$$

where $\mathcal{G}(r)$ is the radial function defined in equation (3.2). Then $P^{m}$ is transient.

\section{Hessian and Laplacian COMParison theory}

We denote by $\nabla^{N} r$ and $\nabla^{P} r$ the gradients of $r$ in $N$ and $P$, respectively. Let us first remark that $\nabla^{P} r(q)$ is just the tangential component in $P$ of $\nabla^{N} r(q)$, for all $q \in P$. Then we have the following basic relation:

$$
\nabla^{N} r=\nabla^{P} r+\left(\nabla^{P} r\right)^{\perp}
$$

where $\left(\nabla^{P} r\right)^{\perp}(q)$ is perpendicular to $T_{q} P$ for all $q \in P$. The unit normal vector field on the boundary of the annulus $\partial A_{\rho, R}=\partial D_{\rho} \cup \partial D_{R}$ is denoted by $\nu$ and defined by the following normalized $P$-gradient of the distance function restricted to $\partial D_{\rho}$ and $\partial D_{R}$, respectively:

$$
\nu=\nabla^{P} r(x) /\left\|\nabla^{P} r(x)\right\|, \quad x \in \partial A_{\rho, R} .
$$

Concerning the 2nd order analysis of $r$ we need firstly and foremost the Hessian comparison theorem for manifolds with a pole:

Theorem 4.1 (See GreW], Theorem A). Let $N^{n}$ be a manifold with a pole $p$, and let $M_{w}^{m}$ denote a $w$-model with center $p_{w}$. Suppose that every $p$-radial sectional curvature at $x \in N-\{p\}$ is bounded from above by the $p_{w}$-radial sectional curvatures in $M_{w}^{m}$ as follows:

$$
K_{p, N}\left(\sigma_{x}\right) \leq-\frac{w^{\prime \prime}(r)}{w(r)}
$$

for every radial two-plane $\sigma_{x} \in T_{x} N$ at distance $r=r(x)=\operatorname{dist}_{N}(p, x)$ from $p$ in $N$. Then the Hessian of the distance function in $N$ satisfies

$$
\begin{aligned}
\operatorname{Hess}^{N}(r(x))(X, X) & \geq \operatorname{Hess}^{M_{w}^{m}}(r(y))(Y, Y) \\
& =\eta_{w}(r)\left(1-\left\langle\nabla^{M} r(y), Y\right\rangle_{M}^{2}\right) \\
& =\eta_{w}(r)\left(1-\left\langle\nabla^{N} r(x), X\right\rangle_{N}^{2}\right)
\end{aligned}
$$

for every unit vector $X$ in $T_{x} N$ and for every unit vector $Y$ in $T_{y} M$ with $r(y)=$ $r(x)=r$ and $\left\langle\nabla^{M} r(y), Y\right\rangle_{M}=\left\langle\nabla^{N} r(x), X\right\rangle_{N}$.

Remark d. Since we shall need this Hessian comparison result below, the above theorem motivates in particular the first assumption in the general comparison constellation of Definition 3.2 We must also note specifically that the theorem holds true with the minimum differentiability assumption for $w(r)$, i.e. with a possibly only piecewise continuous upper bound in the assumption (4.2).

If we let $\mu: N \mapsto \mathbb{R}$ denote a smooth function on $N$, then the restriction $\tilde{\mu}=\mu_{\left.\right|_{P}}$ is a smooth function on $P$ and the respective Hessian operators, $\operatorname{Hess}^{N}(\mu)$ and $\operatorname{Hess}^{P}(\tilde{\mu})$, are related as follows. 
Proposition 4.2 ([JK] $)$.

$$
\begin{aligned}
\operatorname{Hess}^{P}(\tilde{\mu})(X, Y)= & \operatorname{Hess}^{N}(\mu)(X, Y) \\
& +\left\langle\nabla^{N}(\mu), \alpha_{x}(X, Y)\right\rangle
\end{aligned}
$$

for all tangent vectors $X, Y \in T_{x} P^{m} \subset T_{x} N^{n}$, where $\alpha_{x}$ is the second fundamental form of $P$ at $x$ in $N$.

If we modify $\mu$ by a smooth function $f: \mathbb{R} \rightarrow \mathbb{R}$, we then get

Corollary $4.3([\mathrm{JK}])$.

$$
\begin{aligned}
\operatorname{Hess}^{P}( & f \circ \tilde{\mu})(X, X)=f^{\prime \prime}(\mu)\left\langle\nabla^{N}(\mu), X\right\rangle^{2} \\
& +f^{\prime}(\mu)\left(\operatorname{Hess}^{N}(\mu)(X, X)+\left\langle\nabla^{N}(\mu), \alpha_{x}(X, X)\right\rangle\right)
\end{aligned}
$$

for all $X \in T_{x} P^{m}$.

Combining these results and tracing the resulting Hessian comparison statement in an orthornormal basis of $T_{x} P^{m}$, we obtain the following Laplacian inequality:

Proposition 4.4. Suppose again that the assumptions of Theorem 4.1 are satisfied. Then we have for every smooth function $f(r)$ with $f^{\prime}(r) \geq 0$

$$
\begin{aligned}
\Delta^{P}(f \circ r) \geq & \left(f^{\prime \prime}(r)-f^{\prime}(r) \eta_{w}(r)\right)\left\|\nabla^{P} r\right\|^{2} \\
& +m f^{\prime}(r)\left(\eta_{w}(r)+\left\langle\nabla^{N} r, H_{P}\right\rangle\right),
\end{aligned}
$$

where $H_{P}$ denotes the mean curvature vector of $P$ in $N$.

\section{Capacities of (eXtrinsic) annular domains}

We prove Theorem A by showing the existence of a compact subset with positive capacity in $P^{m}$. The compact set in question is constructed as an extrinsic regular ball defined in $P$ using the distance function of the ambient space.

The capacity of a compact domain $K$ in a precompact open set $\Omega$ of a Riemannian manifold $M$ can be expressed as the following integral along the boundary of the compact set $K$ (see e.g. [Gri]):

$$
\operatorname{Cap}(K, \Omega)=\int_{\partial K}\left\langle\nabla^{M} u, \nu\right\rangle d A
$$

where $\nu$ is the unit normal vector field on $\partial K$ which points into the domain $\Omega-K$ and the function $u$ is the solution of the Dirichlet problem in $\Omega-K$

$$
\left\{\begin{aligned}
\Delta^{M} u & =0 \text { on } \Omega-K \\
u & =0 \text { on } \partial K \\
u & =1 \text { on } \partial \Omega
\end{aligned}\right.
$$

In our present setting, the extrinsic ball $D_{R}$ may not be precompact since the submanifold $P$ could contain $N$-bounded but $P$-unbounded sequences of points. Therefore, in order to associate a unique capacity $\operatorname{Cap}\left(D_{\rho}, D_{R}\right)$ to the possibly infinite annular domain $A_{\rho, R}$, we consider any exhaustion sequence of precompact open subsets $\left\{\Sigma_{n}\right\}_{n \in \mathbb{N}}$ covering all of $P^{m}$ such that $\Sigma_{0}=D_{\rho}$ and $\Sigma_{n} \subseteq \Sigma_{n+1}$. Then $\Xi_{n}=\Sigma_{n} \cap D_{R}$ is also an exhaustion sequence for $D_{R}$ by precompact open sets and 
$A_{\rho, R, n}=\Xi_{n}-D_{\rho}$ is likewise an exhaustion sequence for $A_{\rho, R}$ by precompact open sets. The capacity of $A_{\rho, R}$ is then determined by

$$
\begin{aligned}
\operatorname{Cap}\left(A_{\rho, R}\right) & =\operatorname{Cap}\left(D_{\rho}, D_{R}\right) \\
& =\lim _{n \rightarrow \infty} \operatorname{Cap}\left(D_{\rho}, \Xi_{n}\right) \\
& =\lim _{n \rightarrow \infty} \operatorname{Cap}\left(A_{\rho, R, n}\right) .
\end{aligned}
$$

\section{6. $\mathcal{H}$-DRIFTED CAPACITY OF MODEL SPACES}

Definition 6.1. Let $M$ denote a Riemannian manifold with Laplace operator $\Delta^{M}$, and let $V$ denote a continuous vector field on $M$. The drifted Brownian motion on $M$ with drift vector field $V$ is then generated by the linearly modified Laplacian L:

$$
\mathrm{L} f=\Delta^{M} f+\left\langle\nabla^{M} f, V\right\rangle
$$

for every smooth function $f$ on $M$.

We consider in particular the drift vector field $V=-m \mathcal{H}(r) \nabla^{M_{w}^{m}}(r)$ on model spaces $M=M_{w}^{m}$, so that the modified Laplacian then reads for smooth functions $\psi$ on $M_{w}^{m}$ :

$$
\mathrm{L} \psi=\Delta \psi-m \mathcal{H}(r)\langle\nabla \psi, \nabla r\rangle .
$$

For purely radial functions we get

Lemma 6.2. Let $\psi=\psi(r)$ denote a function on the $w$-model space $M=M_{w}^{m}$ which only depends on the radial distance $r$ to the center $p_{w}$. Then

$$
\begin{aligned}
\Delta^{M} \psi(r) & =\psi^{\prime \prime}(r)+(m-1) \eta_{w}(r) \psi^{\prime}(r) \quad \text { and } \\
\mathrm{L} \psi(r) & =\psi^{\prime \prime}(r)+\psi^{\prime}(r)\left((m-1) \eta_{w}(r)-m \mathcal{H}(r)\right) \quad .
\end{aligned}
$$

For convenience and for comparison purposes we introduce the following notations in accordance with Proposition 2.4. The annular domains in the model space are denoted by $A_{\rho, R}^{w}=\left\{x \in M_{w}^{m} \mid \pi(x) \in[\rho, R]\right\}=\pi^{-1}([\rho, R])$, and the corresponding boundaries are denoted by $\partial D_{\rho}^{w}=\pi^{-1}(\rho)$ and $\partial D_{R}^{w}=\pi^{-1}(R)$, respectively. The corresponding Dirichlet problem for the capacity comparison is then

$$
\left\{\begin{array}{rl}
\mathrm{L} \psi & =0 \text { on } A_{\rho, R}^{w}, \\
\psi & =0 \text { on } \partial D_{\rho}^{w} \\
\psi & =1 \text { on } \partial D_{R}^{w}
\end{array},\right.
$$

We express the explicit solution to this problem by means of the exponential integral $\mathcal{G}(r)$ of $\mathcal{H}(r)$ and the warping function $w(r)$ :

Proposition 6.3. The solution to the Dirichlet problem (6.4) only depends on $r$ and is given explicitly by

$$
\psi_{\rho, R}(r)=\left(\int_{\rho}^{r} \frac{\mathcal{G}^{m}(t)}{w^{m-1}(t)} d t\right)\left(\int_{\rho}^{R} \frac{\mathcal{G}^{m}(t)}{w^{m-1}(t)} d t\right)^{-1} .
$$


The corresponding 'drifted' capacity is therefore, in view of equation (5.1),

$$
\begin{aligned}
\operatorname{Cap}_{\mathcal{H}}\left(A_{\rho, R}^{w}\right) & =\frac{\mathcal{G}^{m}(\rho)}{w^{m-1}(\rho)} \operatorname{Vol}\left(\partial D_{\rho}^{w}\right)\left(\int_{\rho}^{R} \frac{\mathcal{G}^{m}(t)}{w^{m-1}(t)} d t\right)^{-1} \\
& =\operatorname{Vol}\left(S_{1}^{m-1}\right)\left(\int_{\rho}^{R} \frac{\mathcal{G}^{m}(t)}{w^{m-1}(t)} d t\right)^{-1} .
\end{aligned}
$$

Proof. Let $\psi$ denote a function of $r$ and let $\Gamma(r)=\psi^{\prime}(r)$. Then according to Lemma 6.2

$$
\begin{aligned}
\mathrm{L} \psi(r) & =\Gamma^{\prime}(r)+\Gamma(r)\left((m-1) \eta_{w}(r)-m \mathcal{H}(r)\right) \\
& =\Gamma^{\prime}(r)+z(r) \Gamma(r)
\end{aligned}
$$

where

$$
\begin{aligned}
-z(r) & =m \mathcal{H}(r)-(m-1) \eta_{w}(r) \\
& =\frac{d}{d r} \ln \mathcal{G}^{m}(r)-\frac{d}{d r} \ln w^{m-1}(r) \\
& =\frac{d}{d r} \ln \left(\frac{\mathcal{G}^{m}(r)}{w^{m-1}(r)}\right) .
\end{aligned}
$$

The general solution to the equation $\mathrm{L} \psi(r)=0$ is determined by

$$
\left\{\begin{array}{l}
\Gamma(r)=C_{1} \exp \left(\int-z(r) d r\right) \quad \text { and } \\
\psi(r)=\int_{\rho}^{r} \Gamma(t) d t+C_{2}
\end{array}\right.
$$

where $C_{1}$ and $C_{2}$ are constants of integration.

Therefore

$$
\psi(r)=C_{1}\left(\int_{\rho}^{r} \frac{\mathcal{G}^{m}(t)}{w^{m-1}(t)} d t\right)+C_{2}
$$

The boundary conditions $\psi(\rho)=0$ and $\psi(R)=1$ are finally satisfied by the following choice of constants:

$$
C_{1}=\left(\int_{\rho}^{R} \frac{\mathcal{G}^{m}(t)}{w^{m-1}(t)} d t\right)^{-1} \quad, \text { and } C_{2}=0 .
$$

It follows that the function $\psi_{\rho, R}(r)$ is indeed a solution to the Dirichlet problem (6.4) as claimed. The maximum principle for the operator $\mathrm{L}$ guarantees that $\psi_{\rho, R}(r)$ is the only solution.

The capacity expressions finally follow from (5.1), from the observation that in a model every fiber $\pi^{-1}(r)$ is a scaled (homothetic) copy of $S_{1}^{m-1}$ - the scaling factor being $w(r)$ - and from the fact that by definition of $\mathcal{G}$ we have $\mathcal{G}(\rho)=1$.

Remark e. Let us note the following analogy in honor of intuition: If the function $\mathcal{H}(r)$ is positive, then the drift vector field $-\mathcal{H}(r) \nabla^{M_{w}^{m}}(r)$ will - in comparison with the non-drifted case - drive the 'current' (or the Brownian motion, or the heat kernel as the case may be) back towards the center $p_{w}$. Intuitively speaking it will thus represent a wind blowing against the process in question, slowing down the motion towards the outer boundary at distance $R$. This corresponds to an increase in the directed effective resistance of the annular region $A_{\rho, R}^{w}$ and hence to a decrease in the directed capacity of the domain as determined by the modified operator L and the Dirichlet problem (6.4). We illustrate this by a simple example. 
Example 6.4. Let $P^{2}$ denote a complete round cylinder of radius $a$ in $N=\mathbb{R}^{3}$. Let $p$ denote a point on the axis of symmetry of $P$. Let $a<\rho<R$ and let $A_{\rho, R}(p)$ denote the corresponding extrinsic annulus of $P$ centered at $p$. Note that $A_{\rho, R}(p)$ has two disjoint components. The total capacity of these components is classically given by

$$
\operatorname{Cap}\left(A_{\rho, R}\right)=\frac{4 \pi a}{L}
$$

where $L$ denotes the length of each cylindrical piece of $A_{\rho, R}$, i.e.,

$$
L=\sqrt{R^{2}-a^{2}}-\sqrt{\rho^{2}-a^{2}} .
$$

In particular, the cylinder is evidently not transient since $\operatorname{Cap}\left(A_{\rho, R}\right) \rightarrow 0$ for $R \rightarrow$ $\infty$. The main point, however, is to observe that the capacity of the finite cylindrical annulus can be estimated from below by means of an $\mathcal{H}$-drifted capacity of a suitable model space annulus. The general precise statement will be stated and proved in the next section. The model space carrier in the present example appears as the third member of a comparison constellation $\left\{P^{2}, \mathbb{R}^{3}, M_{w}^{2}\right\}$ on $[0, R]$, which we now construct and analyze.

We first observe that a mean curvature bounding function $\mathcal{H}(r)$ for the cylinder $P$ in $\mathbb{R}^{3}$ can be given exactly and is best possible by

$$
\mathcal{H}(r)=-\left\langle\nabla^{\mathbb{R}^{3}} r, H_{P}(x)\right\rangle=\frac{1}{2 r}
$$

where the latter equality holds for all $x$ at distance $r$ from $p$. This is due to the rotational symmetry of the cylinder and due to the special choice of $p$. Note that the radius $a$ does not appear in this expression. Then we have

$$
\mathcal{G}(r)=\exp \left(\int_{\rho}^{r} \frac{1}{2 t} d t\right)=\sqrt{\frac{r}{\rho}} .
$$

Secondly, the choice of a warping function $w$ which satisfies the constellation criteria is straightforward. Since the ambient space is $\mathbb{R}^{3}$, we must choose $w(r)$ so that

$$
\left\{\begin{array}{ll}
w(r) & >0 \quad \text { for all } r>0, \\
w(0) & =0, \\
w^{\prime}(0) & =1, \\
w^{\prime}(r) / w(r) & \geq 1 / 2 r \text { for all } r>0 \\
w^{\prime \prime}(r) / w(r) & \leq 0 \quad \text { for all } r>0 .
\end{array},\right.
$$

Evidently there is but one obvious choice for $w$, namely $w(r)=r$. Hence we have the model space: $M_{w}^{2}=\mathbb{R}^{2}$ with the standard flat metric. The corresponding drifted capacity of the model space annulus is now

$$
\begin{aligned}
\operatorname{Cap}_{\mathcal{H}}\left(A_{\rho, R}^{w}\right) & =2 \operatorname{Vol}\left(S_{1}^{1}\right)\left(\int_{\rho}^{R} \frac{\mathcal{G}^{2}(t)}{w(t)} d t\right)^{-1} \\
& =4 \pi\left(\int_{\rho}^{R} \frac{1}{\rho} d t\right)^{-1} \\
& =\frac{4 \pi \rho}{R-\rho} .
\end{aligned}
$$




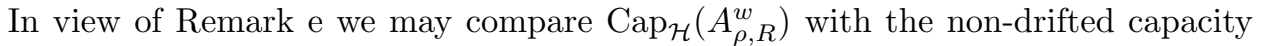
$\operatorname{Cap}_{\mathcal{O}}\left(A_{\rho, R}^{w}\right)$ of the 2-dimensional flat model space annulus (this is again a classical calculation):

$$
\begin{aligned}
\operatorname{Cap}_{\mathcal{O}}\left(A_{\rho, R}^{w}\right) & =2 \operatorname{Vol}\left(S_{1}^{1}\right)\left(\int_{\rho}^{R} \frac{1}{w(t)} d t\right)^{-1} \\
& =4 \pi\left(\int_{\rho}^{R} \frac{1}{t} d t\right)^{-1} \\
& =4 \pi \frac{1}{\ln \left(\frac{R}{\rho}\right)} \\
& >\frac{4 \pi \rho}{R-\rho} \text { for } R>\rho .
\end{aligned}
$$

In accordance with the intuition of Remark $[$ we have thus shown that in this example we have

$$
\operatorname{Cap}_{\mathcal{O}}\left(A_{\rho, R}^{w}\right)>\operatorname{Cap}_{\mathcal{H}}\left(A_{\rho, R}^{w}\right) \text { for all } R>\rho .
$$

However, as mentioned, our main interest is with the comparison of $\operatorname{Cap}_{\mathcal{H}}\left(A_{\rho, R}^{w}\right)$ versus $\operatorname{Cap}\left(A_{\rho, R}\right)$ because we want to estimate the latter from below. Indeed, in this particular example we do get

$$
\operatorname{Cap}\left(A_{\rho, R}\right) \operatorname{Vol}\left(\partial D_{\rho}^{w}\right) \geq \operatorname{Cap}_{\mathcal{H}}\left(A_{\rho, R}^{w}\right) \int_{\partial D_{\rho}}\left\|\nabla^{P} r\right\| d A,
$$

because the left-hand side is

$$
\operatorname{Cap}\left(A_{\rho, R}\right) \operatorname{Vol}\left(\partial D_{\rho}^{w}\right)=\frac{8 \pi^{2} a \rho}{L}=\frac{8 \pi^{2} a \rho}{\sqrt{R^{2}-a^{2}}-\sqrt{\rho^{2}-a^{2}}}
$$

and the right-hand side is

$$
\operatorname{Cap}_{\mathcal{H}}\left(A_{\rho, R}^{w}\right) \int_{\partial D_{\rho}}\left\|\nabla^{P} r\right\| d A=\frac{8 \pi^{2} a}{R-\rho} \sqrt{\rho^{2}-a^{2}}
$$

so that (6.20) follows from a little calculation. The inequality $(6.20)$ is but one illustrating example of our general comparison result which we state and prove in the next section.

\section{THE CAPACITY COMPARISON RESULT}

Our main comparison estimate for the capacities of extrinsic annuli reads as follows:

Theorem 7.1. Let $\left\{N^{n}, P^{m}, M_{w}^{m}\right\}$ denote a comparison constellation on $[0, R]$ in the sense of Definition 3.2. Then

$$
\operatorname{Cap}\left(A_{\rho, R}\right) \operatorname{Vol}\left(\partial D_{\rho}^{w}\right) \geq \operatorname{Cap}_{\mathcal{H}}\left(A_{\rho, R}^{w}\right) \int_{\partial D_{\rho}}\left\|\nabla^{P} r\right\| d A .
$$


Proof. Let us first suppose that $D_{R}$ and hence also $A_{\rho, R}$ is precompact. Let $u$ be the solution of the Dirichlet capacity problem in $A_{\rho, R}$ :

$$
\left\{\begin{aligned}
\Delta^{P} u & =0 \text { on } A_{\rho, R}, \\
u & =0 \text { on } \partial D_{\rho} \\
u & =1 \text { on } \partial D_{R} .
\end{aligned}\right.
$$

Since $\partial D_{\rho}$ is a level surface of $u$ we have that $\nabla^{P} u$ is orthogonal to $\partial D_{\rho}$. On the other hand, $\nu=\nabla^{P} r /\left\|\nabla^{P} r\right\|$ is the unit normal pointing into $A_{\rho, R}$ at the interior boundary $\partial D_{\rho}$ of the annulus. Since $\nabla^{P} u$ is also pointing into $A_{\rho, R}$ we get

$$
\operatorname{Cap}\left(A_{\rho, R}\right)=\int_{\partial D_{\rho}}\left\langle\nabla^{P} u, \nu\right\rangle d A=\int_{\partial D_{\rho}}\left\|\nabla^{P} u\right\| d A .
$$

We now transplant the model space solutions $\psi_{\rho, R}(r)$ of equation (6.4) into the extrinsic annulus $A_{\rho, R}$ in $P$ by defining

$$
\psi_{\rho, R}: A_{\rho, R} \longrightarrow \mathbb{R} ; \quad \psi_{\rho, R}(x)=\psi_{\rho, R}(r(x)) \quad .
$$

Using $w^{\prime}(r)=\eta_{w}(r) w(r)$ and the assumption $\mathcal{H}(r) \leq \eta_{w}(r)$ it is straightforward to check that

$$
\psi_{\rho, R}^{\prime \prime}(r)-\psi_{\rho, R}^{\prime}(r) \eta_{w}(r) \leq 0
$$

Since we also have $\psi_{\rho, R}^{\prime}(r) \geq 0$ we can apply Proposition 4.4 and obtain

$$
\begin{aligned}
\Delta^{P} \psi_{\rho, R} & \geq\left(\psi_{\rho, R}^{\prime \prime}(r)-\psi_{\rho, R}^{\prime}(r) \eta_{w}(r)\right)\left\|\nabla^{P} r\right\|^{2} \\
& +m \psi_{\rho, R}^{\prime}\left(\eta_{w}(r)+\left\langle\nabla^{N} r, H_{P}\right\rangle\right)
\end{aligned}
$$

Using the inequalities

$$
\left\{\begin{array}{l}
\left\langle\nabla^{N} r, H_{P}\right\rangle \geq-\mathcal{H}(r) \quad \text { and } \\
\left\|\nabla^{P} r\right\|^{2} \leq 1
\end{array}\right.
$$

we get

$$
\begin{aligned}
\Delta^{P} \psi_{\rho, R} & \geq\left(\psi_{\rho, R}^{\prime \prime}(r)-\psi_{\rho, R}^{\prime}(r) \eta_{w}(r)\right)+m \psi_{\rho, R}^{\prime}\left(\eta_{w}(r)-\mathcal{H}(r)\right) \\
& =\psi_{\rho, R}^{\prime \prime}(r)+\psi_{\rho, R}^{\prime}(r)\left((m-1) \eta_{w}(r)-m \mathcal{H}(r)\right) \\
& =\mathrm{L} \psi_{\rho, R} \\
& =0 \\
& =\Delta^{P} u .
\end{aligned}
$$

Since $\Delta^{P}\left(\psi_{\rho, R}-u\right) \geq 0$ on $A_{\rho, R}$ and since $\left.\left(\psi_{\rho, R}-u\right)\right|_{\partial A_{\rho, R}}=0$, the Maximum Principle (for $\Delta^{P}$ ) guarantees that

$$
\psi_{\rho, R} \leq u \text { on } A_{\rho, R} .
$$

Furthermore $\left.\psi_{\rho, R}\right|_{\partial D_{\rho}}=\left.u\right|_{\partial D_{\rho}}=0$, so that $\partial D_{\rho}$ is a common level-hypersurface for both the functions $\psi_{\rho, R}(x)$ and $u(x)$. The $P$-gradients of these functions are therefore correspondingly related as follows:

$$
\left\|\nabla^{P} \psi_{\rho, R}(x)\right\| \leq\left\|\nabla^{P} u(x)\right\| \text { for all } x \in \partial D_{\rho} \quad .
$$


Then we have from (7.3) that

$$
\begin{aligned}
\operatorname{Cap}\left(A_{\rho, R}\right) & =\int_{\partial D_{\rho}}\left\|\nabla^{P} u\right\| d A \\
& \geq \int_{\partial D_{\rho}}\left\|\nabla^{P} \psi_{\rho, R}\right\| d A \\
& =\psi_{\rho, R}^{\prime}(\rho) \int_{\partial D_{\rho}}\left\|\nabla^{P} r\right\| d A \\
& =\frac{\operatorname{Cap}_{\mathcal{H}}\left(A_{\rho, R}^{w}\right)}{\operatorname{Vol}_{\partial}\left(\partial D_{\rho}^{w}\right)} \int_{\partial D_{\rho}}\left\|\nabla^{P} r\right\| d A,
\end{aligned}
$$

which finishes the proof of the theorem for precompact extrinsic $R$-balls $D_{R}$. In case $D_{R}$ is not precompact we need to modify the proof using the previously-defined exhaustion sequence $A_{\rho, R, n}$ of $A_{\rho, R}$. For each $n$ we consider the solutions $u_{n}$ and $U_{n}$ to the following well-defined Dirichlet problems on $A_{\rho, R, n}($ compare with (7.2) ):

$$
\begin{aligned}
& \left\{\begin{aligned}
\Delta^{P} u_{n}(x) & =0 \text { for all } x \in A_{\rho, R, n}, \\
u_{n}(x) & =0 \text { for all } x \in \partial D_{\rho}, \\
u_{n}(x) & =\psi_{\rho, R}(x) \text { for all } x \in \partial \Xi_{n}, \text { and }
\end{aligned}\right. \\
& \left\{\begin{aligned}
\Delta^{P} U_{n}(x) & =0 \text { for all } x \in A_{\rho, R, n}, \\
U_{n}(x) & =0 \text { for all } x \in \partial D_{\rho}, \\
U_{n}(x) & =1 \text { for all } x \in \partial \Xi_{n}, \text { respectively. }
\end{aligned}\right.
\end{aligned}
$$

At the outer boundary $\partial \Xi_{n}$ we have $\psi_{\rho, R}(x)=u_{n}(x) \leq 1$ for all $x \in \partial \Xi_{n}$ for all $n$ so that the Maximum Principle guarantees

$$
\psi_{\rho, R}(x) \leq u_{n}(x) \leq U_{n}(x) \text { for all } x \in A_{\rho, R, n} \quad
$$

In consequence we have in particular for all $x \in \partial D_{\rho}$ :

$$
\left\|\nabla^{P} U_{n}(x)\right\| \geq\left\|\nabla^{P} u_{n}(x)\right\| \geq\left\|\nabla^{P} \psi_{\rho, R}(x)\right\| .
$$

Hence we get for every $n$ via precompactness of the associated exhaustion sequence:

$$
\begin{aligned}
\operatorname{Cap}\left(A_{\rho, R, n}\right) & =\int_{\partial D_{\rho}}\left\|\nabla^{P} U_{n}\right\| d A \\
& \geq \int_{\partial D_{\rho}}\left\|\nabla^{P} u_{n}\right\| d A \\
& \geq \int_{\partial D_{\rho}}\left\|\nabla^{P} \psi_{\rho, R}\right\| d A \\
& =\psi_{\rho, R}^{\prime}(\rho) \int_{\partial D_{\rho}}\left\|\nabla^{P} r\right\| d A
\end{aligned}
$$

and therefore

$$
\begin{aligned}
\operatorname{Cap}\left(A_{\rho, R}\right) & =\lim _{n \rightarrow \infty} \operatorname{Cap}\left(A_{\rho, R, n}\right) \\
& \geq \psi_{\rho, R}^{\prime}(\rho) \int_{\partial D_{\rho}}\left\|\nabla^{P} r\right\| d A=\frac{\operatorname{Cap}_{\mathcal{H}}\left(A_{\rho, R}^{w}\right)}{\operatorname{Vol}\left(\partial D_{\rho}^{w}\right)} \int_{\partial D_{\rho}}\left\|\nabla^{P} r\right\| d A,
\end{aligned}
$$

as in (7.10), and this proves the theorem. 


\section{Proof of Theorem A}

Proof of Theorem A. With reference to the Kelvin-Nevanlinna-Royden Criteria alluded to in the Introduction, transience follows from the existence of a compact set $K$ which has strictly positive capacity in $P$. We show that $\operatorname{Cap}\left(D_{\rho}, P^{m}\right)$ is strictly positive for some suitable value of $\rho>0$. By virtue of Theorem 7.1 we have the following inequality:

$$
\begin{aligned}
\operatorname{Cap}\left(D_{\rho}, P^{m}\right) & =\lim _{R \rightarrow \infty} \operatorname{Cap}\left(A_{\rho, R}\right) \\
& \geq \lim _{R \rightarrow \infty}\left(\frac{\operatorname{Cap}_{\mathcal{H}}\left(A_{\rho, R}^{w}\right)}{\operatorname{Vol}\left(\partial D_{\rho}^{w}\right)} \int_{\partial D_{\rho}}\left\|\nabla^{P} r\right\| d A\right) .
\end{aligned}
$$

On the other hand we may choose $\rho$ such that

$$
\int_{\partial D_{\rho}}\left\|\nabla^{P} r\right\| d A>0
$$

Indeed, by assumption, $P$ is unbounded in $N$, i.e. for any radius $r$ the boundary $\partial D_{r}$ is non-empty. Thus for any pair of radii $r_{1}$ and $r_{2}$ there is a smooth curve in $P$ from $\partial D_{r_{1}}$ to $\partial D_{r_{2}}$. Such a curve must contain a point $q$ where the tangent vector has a non-vanishing radial component. At such a point $\nabla^{P} r \neq 0$, so the choice of $\rho=r(q)$ will satisfy equation (8.2). Hence it suffices to show that

$$
\lim _{R \rightarrow \infty} \operatorname{Cap}_{\mathcal{H}}\left(A_{\rho, R}^{w}\right)>0
$$

But this is (via the expression (6.6)) equivalent to the inequality

$$
\lim _{R \rightarrow \infty} \int_{\rho}^{R} \frac{\mathcal{G}^{m}(t)}{w^{m-1}(t)} d t<\infty
$$

and the theorem follows.

\section{Corollaries}

Following Remark a from the Introduction we consider the general problem of extracting suitable transience criteria from Theorem A, but here without stated reference to the choice of auxiliary warping function $w(r)$. In the first corollary below we simply apply the warping function of the space form of a suitable constant negative curvature.

Corollary B. Let $P^{m}(m \geq 2)$ be an unbounded immersed submanifold of an Hadamard-Cartan manifold $N^{n}$ with sectional curvatures $K_{N}$ bounded from above by $b<0$. Suppose that the $p$-radial mean curvature function $H_{p, P}(x)$ of $P^{m}$ in $N^{n}$ from a given point $p$ in $N$ satisfies

$$
H_{p, P}(x) \leq \mathcal{H}_{0}<\frac{m-1}{m} \sqrt{-b}
$$

for some constant $\mathcal{H}_{0}$ and for all $x \in P$ outside some compact set. Then $P^{m}$ is transient. 
Proof. Since we have a constant upper bound on the mean curvatures of $P$ outside a compact set we may choose $\mathcal{H}(r)=\mathcal{H}_{0}$. This gives $\mathcal{G}(r)=\exp \left(\mathcal{H}_{0} r\right)$. Further we may choose $w(r)=Q_{b}(r)=\frac{1}{\sqrt{-b}} \sinh (\sqrt{-b} r)$ stemming from the assumption on the sectional curvatures of $N$. Observe that by the assumption on the $p$-radial mean curvatures of $P$ we have

$$
\begin{aligned}
\mathcal{H}(r) & =\mathcal{H}_{0} \\
& \leq \frac{m-1}{m} \sqrt{-b} \\
& <\sqrt{-b} \\
& <\sqrt{-b} \operatorname{coth}(\sqrt{-b} r)=\frac{w^{\prime}(r)}{w(r)} \text { for all } r .
\end{aligned}
$$

The triple $\left\{N^{n}, P^{m}, M_{w}^{m}\right\}$ is therefore a comparison constellation on $[0, \infty[$ (with the above specific choice of $w$ ). The corollary then follows from Theorem A via the inequalities

$$
\begin{aligned}
\int_{\rho}^{\infty} \frac{\mathcal{G}^{m}(r)}{w^{m-1}(r)} d r & \int_{\rho}^{\infty} \frac{e^{m \mathcal{H}_{0} r}}{\left(\frac{1}{2 \sqrt{-b}}\left(e^{\sqrt{-b} r}-e^{-\sqrt{-b} r}\right)\right)^{m-1}} d r \\
& =\int_{\rho}^{\infty} \frac{e^{m \mathcal{H}_{0} r}}{\left(\frac{1}{2 \sqrt{-b}} e^{\sqrt{-b} r}\left(1-e^{-2 \sqrt{-b} r}\right)\right)^{m-1}} d r \\
& <\int_{\rho}^{\infty} \frac{e^{m \mathcal{H}_{0} r}}{\left(\frac{1}{2 \sqrt{-b}} e^{\sqrt{-b} r}\left(1-e^{-2 \sqrt{-b} \rho}\right)\right)^{m-1}} d r \\
& =\left(\frac{1-e^{-2 \sqrt{-b} \rho}}{2 \sqrt{-b}}\right)^{1-m} \int_{\rho}^{\infty} e^{\left(m \mathcal{H}_{0}-(m-1) \sqrt{-b}\right) r} d r \\
& <\infty
\end{aligned}
$$

The transience in Corollary $\mathrm{B}$ is thus obtained essentially from the convergent integral $\int_{\rho}^{\infty} e^{-t} d t$. The idea of introducing the convergence of $\int_{\rho}^{\infty} t^{-1-\varepsilon} d t$ is immediate and will tentatively reduce the assumption of strictly negative sectional curvatures of the ambient space.

As pointed out by Grigor'yan (via nice examples in Section 15.3 of [Gri]), due to the strong application of the Hessian comparison theorem it is particularly important for the explicit construction of the auxiliary warping function to take account of the radial sectional curvatures of the ambient space even close to the pole, although transience is mainly a feature stemming from the geometry of $P$ in $N$ at 'infinity'. This aspect is therefore specifically addressed in the formulation as well as in the proof of the proposition below, which will serve as the main source for the final corollaries of Theorem A, to be (re-)stated and proved below.

Proposition C. Let $P^{m}$ denote an unbounded submanifold of an ambient manifold $N^{n}$ with pole $p$. Let $\mathcal{H}(r)$ and $\mathcal{K}(r)$ denote radial bounding functions for the mean 
curvatures of $P$ and for the p-radial sectional curvatures of $N$, respectively (as in Theorem A). Suppose that for some $\varepsilon>0$ and for some $\rho>0$ we have

$$
\left\{\begin{array}{cl}
-(1+\varepsilon) / r & \leq \mathcal{H}(r) \quad \text { for all } r \geq \rho, \text { and } \\
b & \geq \mathcal{K}(r) \quad \text { for all } r \leq \rho,
\end{array}\right.
$$

where $b$ is the unique real constant satisfying the following transcendental equation:

$$
\eta_{Q_{b}}(\rho)=\frac{1+\varepsilon}{\rho(m-1)}+\frac{m}{m-1} \mathcal{H}(\rho)
$$

(i.e. $b$ is the constant curvature of the unique space form whose distance sphere of radius $\rho$ has its mean curvature equal to the right-hand side of equation (9.5)). Suppose further that $\mathcal{H}(r)$ is differentiable for all $r \geq \rho$, and that

$$
\left\{\begin{array}{c}
(1+\varepsilon)(m-2-\varepsilon)\left(\frac{1}{r^{2}}\right) \\
-2 m(1+\varepsilon)\left(\frac{1}{r}\right) \mathcal{H}(r) \\
-m(m-1) \mathcal{H}^{\prime}(r) \\
-m^{2} \mathcal{H}^{2}(r) \\
\geq(m-1)^{2} \mathcal{K}(r) \quad \text { for all } r \geq \rho
\end{array}\right.
$$

Then $P^{m}$ is transient.

Proof. We first define a tentative candidate $\mu(r)$ for the warping function $w(r)$ for $r \geq \rho$. Let $\mu(r)$ denote the following function:

$$
\mu(r)=r^{\alpha} \mathcal{G}^{\beta}, \quad \text { where } \quad \alpha=\frac{1+\varepsilon}{m-1} \quad \text { and } \quad \beta=\frac{m}{m-1} \quad .
$$

Then

$$
\int_{\rho}^{\infty} \frac{\mathcal{G}^{m}(r)}{\mu^{m-1}(r)} d r=\int_{\rho}^{\infty} \frac{1}{r^{1+\varepsilon}} d r<\infty
$$

so that $P$ is transient, if we use $w(r)=\mu(r)$ and if we can satisfy the curvature conditions of Theorem A. To that end we first have for $r \geq \rho$ :

$$
\frac{\mu^{\prime}(r)}{\mu(r)}=\frac{\alpha}{r}+\beta \frac{\mathcal{G}^{\prime}(r)}{\mathcal{G}(r)}=\frac{\alpha}{r}+\beta \mathcal{H}(r)
$$

so that

$$
\frac{\mu^{\prime}(r)}{\mu(r)} \geq \mathcal{H}(r) \quad \text { if and only if } \quad(\beta-1) \mathcal{H}(r) \geq-\frac{\alpha}{r}
$$

but this follows (with $w(r)=\mu(r)$ ) from the first condition in (9.4). Correspondingly we have

$$
\begin{aligned}
\frac{\mu^{\prime \prime}(r)}{\mu(r)}= & \alpha(\alpha-1)\left(\frac{1}{r^{2}}\right)+2 \alpha \beta\left(\frac{1}{r}\right) \mathcal{H}(r) \\
& +\beta^{2} \mathcal{H}^{2}(r)+\beta \mathcal{H}^{\prime}(r),
\end{aligned}
$$

so that the condition $-\mu^{\prime \prime}(r) / \mu(r) \geq \mathcal{K}(r)$ is, when $w(r)=\mu(r)$, equivalent to (9.6). Finally we must prolong $\mu(r)$ into the interval $[0, \rho]$ and guarantee that the curvature condition is also satisfied there as well as the initial conditions for the warping function. We tentatively define

$$
\xi(r)=Q_{b}(r) \frac{\mu(\rho)}{Q_{b}(\rho)} \quad \text { for } r \leq \rho
$$


The function $\xi(r)$ is indeed a $C^{1}$ extension of $\mu(r)$, since we have by choice of $b$ that

$$
\xi(\rho)=\mu(\rho) \quad \text { and } \quad \frac{\xi^{\prime}(\rho)}{\xi(\rho)}=\frac{\mu^{\prime}(\rho)}{\mu(\rho)} .
$$

We observe that the necessary curvature inequality is also satisfied for this extension, since we have by assumption

$$
-\frac{\xi^{\prime \prime}(r)}{\xi(r)}=b \geq \mathcal{K}(r) \quad \text { for all } r \in[0, \rho]
$$

At this point we then consider the following warping function $w(r)$ which inherits the curvature inequalities from $\xi(r)$ and $\mu(r)$ and which still makes the integral in (9.8) converge:

$$
w(r)= \begin{cases}\mu(r) / \xi^{\prime}(0) & \text { for } r \geq \rho \text { and } \\ \xi(r) / \xi^{\prime}(0) & \text { for } r \leq \rho .\end{cases}
$$

Moreover, $w(r)$ satisfies the initial conditions at 0 . This warping function could possibly have a discontinuous relative second order derivative at $\rho$. However, as already mentioned, the Hessian comparison theorem and its consequences still hold true for radial curvature functions with a finite number of discontinuities, so we conclude from Theorem A that $P$ is transient.

In order to put special emphasis on the interplay between the intrinsic geometric conditions and the extrinsic geometric conditions for transience - as was requested and alluded to in the Introduction - we now apply Proposition $\mathrm{C}$ to 3 examples. In each case we impose a strong condition in one of the following 'directions': Strong extrinsic geometric conditions on the submanifold, strong intrinsic geometric conditions on the ambient space, and finally the case of coalescence $P=N$ (where the extrinsic geometry disappears from the analysis and only the intrinsic geometry of $N$ is active).

9.1. Concerning the submanifold. If the submanifold is known to posses some given strong geometric property, then we may ask for conditions on the ambient space which will imply transience of the submanifold. From this viewpoint we consider minimal submanifolds and radial cones. These latter objects are defined as follows.

Definition 9.1. Let $Q^{m-1}$ denote a compact smooth submanifold of dimension $m-1$ in an $m$-dimensional linear affine subspace in $\mathbb{R}^{n}$, which does not contain the origin. The Euclidean cone $C_{Q}^{m}$ over $Q$ in $\mathbb{R}^{n}$ is then the cone generated by the straight lines through the origin and through the points of $Q$. A $p$-radial cone in a manifold $N^{n}$ with a pole $p$ is the image $\exp _{p} C_{Q}^{m}$ of a Euclidean cone $C_{Q}^{m}$ in $T_{p} N$.

Observation 9.2. Let $P^{m}$ denote an $m$-dimensional submanifold in an ambient manifold $N^{n}$ with a pole $p$. Suppose that $P^{m}$ is either a p-radial cone or a minimal submanifold. Then the p-radial mean curvature function for $P^{m}$ vanishes identically, and it is therefore bounded from above by $\mathcal{H}(r)=0$ for all $r=r(x), x \in P$. 
Thus, if we insert $\mathcal{H}(r)=0$ in Proposition $\mathrm{C}$ we get:

Corollary D. Let $P^{m}$ denote an unbounded $m$-dimensional submanifold in an ambient manifold $N^{n}$ with a pole $p$. Suppose that $P$ is either a p-radial cone or a minimal submanifold outside some compact subset in $N$. Then $P^{m}$ is transient if the p-radial sectional curvatures of $N$ are bounded from above as follows (for some $\varepsilon>0$ and some $\rho>0)$ :

$$
\left\{\begin{array}{l}
\mathcal{K}(r) \leq(1+\varepsilon)(m-2-\varepsilon) /((m-1) r)^{2} \quad \text { for } \quad r \geq \rho, \\
\mathcal{K}(r) \leq b \text { for } r \leq \rho,
\end{array}\right.
$$

where $b$ is the solution to $\eta_{Q_{b}}(\rho)=(1+\varepsilon) /(\rho(m-1))$.

In particular for $m=2$ we have

Corollary E. Suppose $P^{2}$ is an unbounded 2-dimensional surface in a manifold $N^{n}$ with a pole $p$. Suppose that (outside the metric sphere of radius $\rho$ and center $p$ in $N$ ) the surface $P$ is either a p-radial cone or a minimal surface. Suppose further that there exists a constant $\varepsilon>0$, such that the p-radial sectional curvatures of $N$ are less than or equal to $-\varepsilon(1+\varepsilon) / r^{2}$ for all $r \geq \rho$ and less than or equal to $b(\varepsilon, \rho)$ for all $r \leq \rho$, where $b(\varepsilon, \rho)$ is the (negative) solution to the equation

$$
\operatorname{coth}(\rho \sqrt{-b(\varepsilon, \rho)})=(1+\varepsilon) /(\rho \sqrt{-b(\varepsilon, \rho)}) .
$$

Then $P^{2}$ is transient.

Proof. We only need to put $m=2$ in the previous corollary and observe that the constant $b$ is now necessarily negative because $(1+\varepsilon) / \rho>1 / \rho$ and $\eta_{Q_{b}}(r) \leq 1 / r$ for all $b \geq 0$ and for all $r>0$.

9.2. Concerning the ambient space. If the ambient space is a model space or some other space with strong geometric properties, then we may ask for sufficient conditions on the submanifold which will imply transience of the submanifold. For example, considering flat Euclidean ambient spaces we have:

Corollary F. Let $P^{m}$ denote an unbounded submanifold in $\mathbb{R}^{n}$. Let $\mathcal{H}(r)$ denote a smooth bounding function for the $p$-radial mean curvatures of $P$ for some $p \in \mathbb{R}^{n}$. Suppose that for some $\varepsilon>0$ and for some $\rho>0$ we have

$$
\left\{\begin{array}{rl}
-(1+\varepsilon) / r \leq \quad & \mathcal{H}(r) \quad \text { for all } r \geq \rho, \text { and } \\
& \mathcal{H}(\rho) \leq(m-2-\varepsilon) /(m \rho)
\end{array} .\right.
$$

Suppose further that

$$
\left\{\begin{array}{c}
(1+\varepsilon)(m-2-\varepsilon)\left(\frac{1}{r^{2}}\right) \\
-2 m(1+\varepsilon)\left(\frac{1}{r}\right) \mathcal{H}(r) \\
-m(m-1) \mathcal{H}^{\prime}(r) \\
-m^{2} \mathcal{H}^{2}(r) \\
\geq 0 \text { for all } r \geq \rho .
\end{array}\right.
$$

Then $P^{m}$ is transient.

Proof. This follows directly from Proposition C upon observing that the second inequality in (9.17) guarantees that condition (9.5) is satisfied by some $b \geq 0$ so that all of (9.4) holds with $\mathcal{K}(r)=0$. 
For 2-dimensional submanifolds $P^{2}$ in $\mathbb{R}^{n}$ the conditions of Corollary $\mathrm{E}$ are particularly difficult to satisfy. We suspect that there is in fact no such surface which can satisfy these conditions. This is in part motivated by the following:

Observation 9.3. Let $P^{2}$ denote a 2-dimensional submanifold in $\mathbb{R}^{n}$ which satisfies all the conditions of Corollary $\mathbb{E}$. Then the following inequality is also satisfied for all $r=r(x) \geq \rho, x \in P$ :

$$
\left\langle\nabla^{P} r, H_{P}(x)\right\rangle \geq \varepsilon / 2 r
$$

In other words, the surface is everywhere curved (in the mean) away from the point $p$ by the same amount (modulo the scaling by $\varepsilon / 2$ ) as the distance spheres are curved towards $p$.

Proof. Suppose the conditions of Corollary [E are satisfied. Then (9.18) specializes to

$$
\mathcal{H}^{\prime}(r) \leq-\frac{1}{2}\left(\varepsilon(1+\varepsilon) r^{-2}+4(1+\varepsilon) r^{-1} \mathcal{H}(r)+4 \mathcal{H}^{2}(r)\right) .
$$

The inequality $\mathcal{H}(r) \leq-\varepsilon / 2 r$ is satisfied at $r=\rho$ because of (9.17). We now show that $\mathcal{H}(r)<-\kappa \varepsilon / 2 r$ for every $\kappa<1$ and all $r \geq \rho$. Then (9.19) follows by letting $\kappa \rightarrow 1$. Suppose for contradiction that for some smallest value $r_{0}>\rho$ we have $\mathcal{H}\left(r_{0}\right)=-\kappa \varepsilon / 2 r_{0}$ for some $\kappa<1$. Then the derivative at $r_{0}$ of $\mathcal{H}(r)$ must be larger than or equal to $\kappa \varepsilon / 2 r_{0}^{2}$. In consequence we have from (9.20):

$$
\kappa \varepsilon \leq-\left(\varepsilon(1+\varepsilon)-2 \kappa \varepsilon(1+\varepsilon)+\kappa^{2} \varepsilon^{2}\right) \quad \Leftrightarrow \quad \varepsilon(1-\kappa) \leq-1,
$$

which is the desired contradiction.

Remark f. This observation is but one indication (out of several others, see e.g. [MM]) that the problem of finding extrinsic geometric hypotheses which will induce transience for submanifolds in ambient Riemannian spaces is still quite challenging for classical 2-dimensional surfaces in $\mathbb{R}^{n}$ and even for surfaces in 3-dimensional Euclidean space.

9.3. Concerning the purely intrinsic viewpoint. We show how the well-known transience result of Ichihara may be obtained by specializing our proof of Theorem A to the purely intrinsic setting of a given manifold $N$ with a pole.

Proposition G ([1], [12] $)$. Let $N^{n}$ denote a complete Riemannian manifold with a pole $p$. Suppose that the p-radial sectional curvatures of $N^{n}$ are bounded from above by the $p_{w}$-radial sectional curvatures of a $w$-model space $M_{w}^{m}$ for all $r>0$. If furthermore the following condition holds:

$$
\int_{\rho}^{\infty} \frac{1}{w^{n-1}(r)} d r<\infty
$$

then $N^{n}$ is transient.

Proof. This is not a direct consequence of our main theorem, but rather a corollary of its proof. Indeed, when $P^{m}=N^{n}$ we have the equality $\left\|\nabla^{P} r\right\|=1$, and then (7.5) is obsolete for the statements of (7.8) to hold true. In consequence we do not need the assumption that $\mathcal{H}(r) \leq \eta_{w}(r)$. Moreover, in the Laplacian comparison analysis we may formally put $H_{P}=0$, since $P=N$. The conclusion follows as before, but now from the sole assumption that the $p$-radial sectional curvatures of $N$ are bounded from above by the $p_{w}$-radial sectional curvatures of the comparison model space. 


\section{REFERENCES}

[GreW] R. Greene and H. Wu, Function theory on manifolds which possess a pole, Lecture Notes in Math., vol. 699, Springer-Verlag, Berlin and New York (1979). MR0521983 (81a:53002)

[Gri] A. Grigor'yan, Analytic and geometric background of recurrence and non-explosion of the Brownian motion on Riemannian manifolds, Bull. Amer. Math. Soc. 36 (1999), 135-249. MR.1659871 (99k:58195)

[H] I. Holopainen, Volume growth, Green's functions and parabolicity of ends, Duke Math. J. 97 (1999), 319-346. MR 1682233 (2000i:58066)

[I1] K. Ichihara, Curvature, geodesics and the Brownian motion on a Riemannian manifold I; Recurrence properties, Nagoya Math. J. 87 (1982), 101-114. MR0676589(84m:58166a)

[I2] K. Ichihara, Curvature, geodesics and the Brownian motion on a Riemannian manifold II; Explosion properties, Nagoya Math. J. 87 (1982), 115-125. MR0676590|(84m:58166b)

[JK] L. P. Jorge and D. Koutroufiotis, An estimate for the curvature of bounded submanifolds, Amer. J. Math. 103 (1981), 711-725. MR0623135(83d:53041b)

[LS] T. Lyons and D. Sullivan, Function theory, random paths and covering spaces, J. Diff. Geometry 19 (1984), 299-323. MR0755228 (86b:58130)

[M] S. Markvorsen, On the mean exit time from a minimal submanifold, J. Diff. Geom. 29 (1989), 1-8. MR0978073 (90a:58190)

[MM] S. Markvorsen and M. Min-Oo, Global Riemannian Geometry: Curvature and Topology, Advanced Courses in Mathematics; CRM Barcelona, Birkhäuser (2003). MR2003861 (2004d:53001)

[MP1] S. Markvorsen and V. Palmer, Generalized isoperimetric inequalities for extrinsic balls in minimal submanifolds, J. reine angew. Math. 551 (2002), 101-121. MR1932175 (2003k:53072)

[MP2] S. Markvorsen and V. Palmer, Transience and capacity of minimal submanifolds, GAFA, Geometric and Functional Analysis 13 (2003), 915-933. MF 2006562

[O'N] B. O'Neill, Semi-Riemannian Geometry; With Applications to Relativity, Academic Press (1983). MR0719023 (85f:53002)

[P] V. Palmer, Isoperimetric inequalities for extrinsic balls in minimal submanifolds and their applications, J. London Math. Soc. 60 (2) (1999), 607-616. MR.1724821 (2000j:53050)

Department of Mathematics, Technical University of Denmark, DK-2800 Kgs Lyngby, DENMARK

E-mail address: S.Markvorsen@mat.dtu.dk

Departament de Matemàtiques, Universitat Jaume I, 12071 Castellon, Spain

E-mail address: palmer@mat.uji.es 